\title{
Dickkopf-3 is regulated by the MYCN-induced miR-17-92 cluster in neuroblastoma
}

\author{
Sara De Brouwer ${ }^{1}$, Pieter Mestdagh ${ }^{1}$, Irina Lambertz ${ }^{1}$, Filip Pattyn ${ }^{1}$, Anne De Paepe ${ }^{1}$, Frank Westermann ${ }^{2}$, \\ Christina Schroeder ${ }^{2}$, Johannes H. Schulte ${ }^{3}$, Alexander Schramm ${ }^{3}$, Katleen De Preter ${ }^{1}$, \\ Jo Vandesompele ${ }^{1}$ and Frank Speleman ${ }^{1}$ \\ ${ }^{1}$ Center for Medical Genetics, Ghent University Hospital, Ghent, Belgium \\ ${ }^{2}$ Department of Tumor Genetics, German Cancer Research Center, Heidelberg, Germany \\ ${ }^{3}$ Division of Hematology and Oncology, University of Children's Hospital, Essen, Germany
}

Neuroblastoma (NB) is a paediatric tumour with a remarkable diverse clinical behaviour. Approximately half of the high stage aggressive tumours are characterized by MYCN gene amplification but our understanding of the role of MYCN in NB oncogenesis is incomplete. Previous studies have shown that MYCN expression is inversely correlated with expression of Dickkopf-3 (DKK3), a gene encoding an extracellular protein with presumed tumour suppressor activity, but direct MYCN regulation of $D K K 3$ was excluded leaving the mechanism of regulation unexplained. Given the recently established role of MYCN-regulated miRNAs in downregulation of protein-coding genes and predicted seeds for miR-17-92 cluster members within the DKK3 $3^{\prime}$ UTR, we hypothesized that this mechanism would act in MYCN regulation of DKK3. To investigate this, we used a validated miR-17-92-inducible cellular system and could demonstrate robust downregulation of DKK3 mRNA and protein levels upon miR-17-92 overexpression. Next, two of the three predicted miRNAs, miR-19b and miR-92a, were shown to lower DKK3 protein levels, in addition to measurable DKK3 mRNA knock-down by miR-92a. Direct interaction between miR-19b or miR-92a and the $3^{\prime} U T R$ of $D K K 3$ was validated using luciferase reporter assays. In conclusion, this study demonstrates that the MYCN-induced downregulation of DKK3 results from direct upregulation of miR-17-92 components effecting both DKK3 mRNA stability and translation which further contributes to the pleiotropic oncogenic effect of elevated MYCN levels. The strict MYCN-mediated regulation of DKK3 is suggestive for an important downstream function of the MYCN protein and thus warrants further investigations to unravel the role of DKK3 in NB.

Neuroblastoma $(\mathrm{NB})$ is a paediatric tumour with a worldwide incidence of 10 cases per million in children younger than 15 years old. ${ }^{1} \mathrm{NB}$ originates from sympathetic neuronal progenitors and is characterized by a remarkable diverse clinical behaviour ranging from spontaneously regressing to highly aggressive metastasized tumours. Amplification of MYCN occurs with a high incidence in aggressive tumour phenotype and, furthermore, MYCN status can be used as an independent predictor of poor prognosis. MYCN is member of the

Key words: neuroblastoma, dickkopf-3, miR-17-92 cluster Additional Supporting Information may be found in the online version of this article.

Grant sponsors: Flemish League against Cancer, Belgian Program of Interuniversity Poles of Attraction, initiated by the Belgian State, Prime Minister's Office, Science Policy Programming, Fund for Scientific Research; Grant numbers: G.O198.08, 01G01910

DOI: $10.1002 /$ ijc. 26295

History: Received 3 Mar 2011; Accepted 24 Jun 2011; Online 27 Jul 2011

Correspondence to: Frank Speleman, Center for Medical Genetics, Ghent University Hospital, De Pintelaan 185, B-9000 Ghent,

Belgium, Tel.: +32-9-332-2451, Fax: +32-9-332-6549,

E-mail: franki.speleman@UGent.be
MYC family of transcription factors which contain a basic helix-loop-helix leucine zipper (HLH-zip) domain that is important for protein dimerization, sequence specific DNA binding and regulation of transcription. ${ }^{2,3}$ Evidence for a direct role for $\mathrm{MYCN}$ in $\mathrm{NB}$ development was obtained through the generation of a transgenic mouse model where a tyrosine hydroxylase-driven overexpression of MYCN in sympathetic neural crest progenitor cells resulted in the development of NB tumours. ${ }^{4}$ Subsequently, further studies aimed at identifying the transcriptional targets of MYCN in NB cells by using $M Y C N$-regulable constructs, ${ }^{5-7}$ retinoic acid treatment ${ }^{8,9}$ and siRNA-mediated MYCN knockdown. ${ }^{10-12}$ Remarkably, despite these in-depth investigations, only a limited number of bona fide upregulated target genes could be identified, including ODC1, MDM2, MCM7 and hTERT. ${ }^{5,13-15}$ Transcriptional upregulation results from binding of the MYCN/MAX heterodimer to E-box sequences in the promoter region of the target genes as well as through binding to non-canonical sequences. ${ }^{16}$ In addition to activation of transcription, MYCN can also function as a transcriptional repressor of genes such as TRKA and p75NTR. Here, MYCN associates with transcription factors MIZ1/SP1 followed by a recruitment of HDAC1, thereby inducing a repressed chromatin state. ${ }^{17}$ These and other studies indicate that MYCN controls a broad regulatory network implicated 
in cell cycle, DNA damage response, differentiation and apoptosis. Unravelling the downstream pathways controlled by MYCN is essential for understanding how this transcription factor contributes to the process of tumour development, particularly that of $\mathrm{NB}$, which will further be important in the choice of appropriate therapeutic targets.

In addition to the above-mentioned direct MYCN targets, the Dickkopf-3 (DKK3) gene has also been studied as it appears to be consistently regulated in several systems upon modulation of $M Y C N$ expression. ${ }^{18}$ DKK3 is a member of the DKK family of proteins which play important roles in normal development and in the negative modulation of the $\mathrm{Wnt} / \beta$-catenin signaling pathway. ${ }^{19-22}$ High expression levels of this putative tumour suppressor gene was shown to be positively correlated with good prognosis for NB patients, ${ }^{23,24}$ whereas reduced DKK3 expression has been observed in multiple cell lines and tumours of endothelial and epithelial origin. In certain tumour entities, decreased DKK3 expression levels were found in association with promoter-hypermethylation. ${ }^{23,25-28}$ Furthermore, overexpression of DKK3 was shown to inhibit proliferation and motility both in vitro and in vivo. ${ }^{2529-36}$ Taken together, these data are in keeping with a tumour suppressor function for DKK3.

Koppen and colleagues observed that DKK3 mRNA expression in NB cell lines was negatively correlated with MYCN expression levels and further demonstrated MYCN-mediated downregulation of $D K K 3$ using a MYCN-inducible cellular system. Chromatin immunoprecipitation assays, however, failed to identify direct interaction between MYCN and the promoter region of the DKK3 gene, implying a mechanism of regulation different from direct transcriptional inhibition. ${ }^{23}$

Recently, several studies have demonstrated that an indirect MYCN-mediated downregulation of gene expression results from direct activation of specific microRNAs (miRNAs). ${ }^{6,37}$ MiRNAs are small non-coding RNAs that negatively regulate the expression of target mRNAs at post-transcriptional level and they are known important regulators of oncogenes and tumour suppressor genes. In a previous study, we explored the role of the MYCN-driven oncogenic miR-17-92 cluster in NB cells. MiR-17-92 activation was shown to result in a profound downregulation of TGF- $\beta$ pathway activity, accompanied by increased cell proliferation in vitro and in vivo. Moreover, high expression of the miR-17-92 cluster in NB tumours was significantly correlated to poor patient survival. ${ }^{38}$ Since DKK3 is indirectly downregulated by $\mathrm{MYCN}$, we hypothesized that $D K K 3$ translational repression and/or degradation could be indirectly controlled through the MYCN-activated miR-17-92 cluster. In this paper, we confirmed a direct regulation of both DKK3 mRNA and DKK3 protein levels by miR-17-92 components, thus providing a mechanistic basis for the tight downregulation of DKK3 levels through MYCN in NB cells.

\section{Material and Methods \\ Samples and cell lines}

A representative cohort of 101 primary untreated NB tumours of all clinical stages was used for analysis. Written informed consent was obtained from the patients' relatives and an ethical approval from the Ghent University Hospital Ethical Committee (EC2008/159) was also obtained.

The SHEP-Tet $21 \mathrm{~N}$ cell line is derived from the SHEP NB cell line and contains a MYCN tet-off system where, more specifically, the expression of a MYCN construct is repressed by tetracycline in the culture medium. The SHEP-TR-miR17-92 NB cell line contains a tet-on system to induce the oncogenic miR-17-92 cluster with the addition of $2 \mu \mathrm{g} / \mathrm{ml}$ of tetracycline to the culture medium. In the SHEP MYCN-ER cell line, the cDNA of MYCN is fused to a mutated estrogen responsive domain (ER) which can bind 4-hydroxy-tamoxifen (4-OHT) but is unable to bind with natural estrogen. ${ }^{6}$ Addition of $200 \mathrm{nM} 4$-OHT to the culture medium activates the MYCN-ER expression.

Data from the NCI-60 cell line panel was downloaded from the National Cancer Institute's Developmental Therapeutic Program Web site (www.sanger.ac.uk/genetics/CGP/ NCI60/).

\section{Cell culture and transfection}

All NB cell lines were cultured in RPMI1640 medium (Invitrogen) supplemented with 10\% FBS, 1\% PEN/STREP, $1 \%$ kanamycin and $1 \%$ glutamine at $37^{\circ} \mathrm{C}$ in a $5 \% \mathrm{CO}_{2}$ atmosphere. DLD1Dicer ${ }^{\text {hypo }}$ cells were grown in DMEM medium (Invitrogen) using the same supplements as the RPMI1640 medium.

SHEP cells were seeded at 150,000 cells per 6-well, $24 \mathrm{hr}$ prior to transfection. Transfection was performed using the XtremeGene lipid (Roche) mixed with OPTI-MEM (Invitrogen) and $100 \mathrm{nM}$ pre-miR (-19a, 19b, -92a or non-targeting control). Conditioned medium was harvested for ELISA and cells were pelleted for RNA isolation (miRNeasy mini kit, Qiagen) and for subsequent DKK3 expression analysis $24 \mathrm{hr}$, 48 and $72 \mathrm{hr}$ post-transfection.

SHEP MYCN-ER cells were seeded at 150,000 cells per 6well, $24 \mathrm{hr}$ prior to 4 -OHT induction. Transfection was performed $24 \mathrm{hr}$ post 4-OHT induction using the XtremeGene lipid (Roche) mixed with OPTI-MEM (Invitrogen) and $100 \mathrm{nM}$ anti-miR (-92a or non-targeting control). Cells were pelleted for RNA isolation $48 \mathrm{hr}$ post-transfection.

\section{Quantative polymerase reaction (qPCR)}

cDNA synthesis was performed on $2 \mu \mathrm{g}$ total RNA (isolated using the miRNeasy mini-kit, Qiagen according to the manufacturer's protocol) with $4 \mu \mathrm{l}$ of iScript reaction mix and $1 \mu \mathrm{l}$ of iScript reverse transcriptase (iScript, Bio-Rad) in a final volume of $20 \mu \mathrm{l}$. Subsequently, this mix was incubated for $5^{\prime}$ at $25^{\circ} \mathrm{C}, 30^{\prime}$ at $42^{\circ} \mathrm{C}$ and $5^{\prime}$ at $85^{\circ} \mathrm{C}$ using the iCycer (Bio$\mathrm{Rad})$. The expression levels were analyzed using $5 \mathrm{ng}$ cDNA, $2.5 \mu \mathrm{l}$ of SYBR Green I mastermix (Eurogentec) and $5 \mu \mathrm{M}$ primers in a total volume of $5 \mu \mathrm{l}$. Expression levels were normalized against two of four reference gene (GAPDH, HPRT1, $Y W H A Z, S D H A)$ and analyzed using the qBasePlus software (http://www.biogazelle.com). All used qPCR assays are 


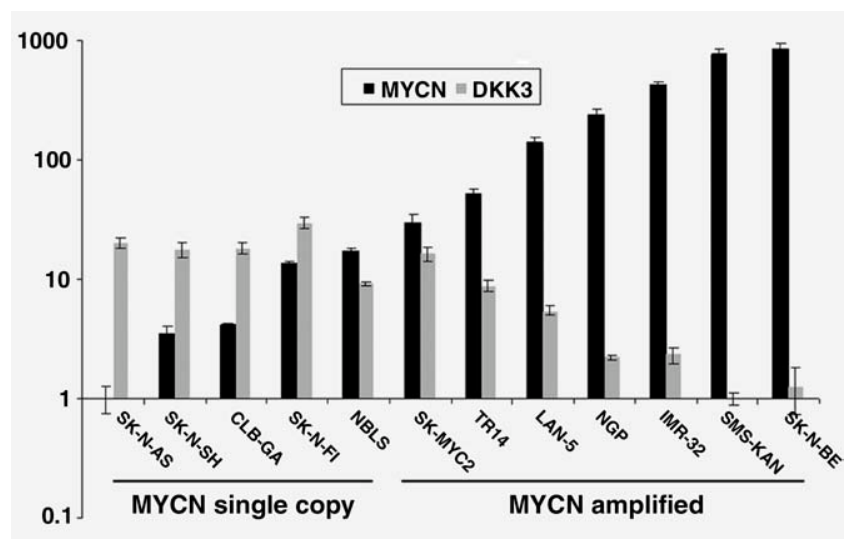

Figure 1. Inverse correlation of $D K K 3$ and $M Y C N$ in a series of NB cell lines. In cell lines with MYCN amplification (SK-MYC2, TR14, LAN-5, NGP, IMR-32, SMS-KAN, SK-N-BE), the expression of DKK3 is low while $D K K 3$ expression is high in cell lines that are $M Y C N$ single copy (SK-N-AS, SK-N-SH, CLB-GA, SK-N-FI, NBLS). The $y$-axis is shown in logarithmic scale.

available in RTPrimerDB with IDs 3 (GAPDH), 5 (HPRT1), 9 (YWHAZ), 7 (SDHA), 8229 (DKK3) and 180 (MYCN) (http://www.rtprimerdb.org). ${ }^{39}$

\section{In silico miRNA target predictions}

MiRNAs putatively targeting the $3^{\prime}$ UTR of DKK3 were investigated using the online prediction databases TargetScan v5.1 (http://www.targetscan.org) and DIANA microT v3.0 (http:// diana.cslab.ece.ntua.gr/microT/).

\section{Luciferase assay}

The $3^{\prime}$ UTR of the human DKK3 gene was cloned in a vector containing the firefly luciferase open reading frame (pSGG_3'UTR, SwitchGear Genomics). Seed regions were mutated using the QuickChange II mutagenesis kit (Stratagene). Specifically, the miR-19 complementary site, gTtacCA, was changed into tTgcaCA and the miR-92a complementary site, gTgcaATA, was changed into tTtacATA (mutated sites in lower case). Prior to seeding of the cells, plates were coated with fibronectin (Tebu-Bio) for a better attachment of the DLD1Dicer ${ }^{\text {hypo }}$ cells. Cells were plated $24 \mathrm{hr}$ prior to transfection at 10,000 cells per 96-well in DMEM medium containing $10 \%$ FBS. Transfection mixes contained RPMI1640, 50 nM pre-miR (Applied Biosystems), $0.4 \%$ DharmaFect Duo, $100 \mathrm{ng} 3^{\prime}$ UTR vector and $20 \mathrm{ng}$ pRL-TK vector, containing the Renilla luciferase (Promega). The ratios between firefly and Renilla luciferase were measured $48 \mathrm{hr}$ post-transfection using the dual-glo luciferase kit (Promega) according to the manufacturer's protocol.

\section{Enzyme-linked immunosorbent assay (ELISA)}

Cell culture supernatants were collected at various time points and cleared of cellular debris by microcentrifugation. The concentration of secreted DKK3 was measured using the human Dkk-3 DuoSet ELISA kit (R\&D Systems, DY1118)

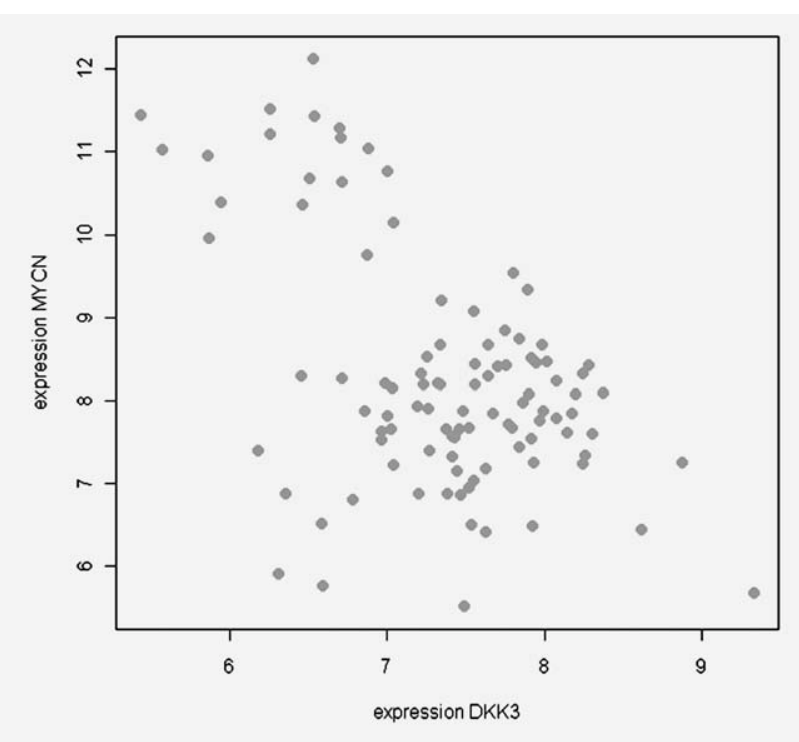

Figure 2. Correlation of DKK3 and MYCN expression in a series of 101 primary untreated NB tumours. Expression of DKK3 ( $x$-axis) and MYCN (y-axis) are significantly inversely correlated in $101 \mathrm{NB}$ tumours (Spearman correlation $r=-0.30, p<0.01$ ).

according to the manufacturer's instructions. Optical density was measured using a Multiskan RC plate reader (LabSystems) and values were plotted against a 4-PL standard curve (Genesis software). Finally, DKK3 concentration values were normalized against total micrograms of protein in the cell culture supernatants, determined by the DC protein assay (Biorad, 500-0116).

\section{Statistical analysis}

Correlation analysis of gene expressions in 101 tumour samples was evaluated with the Spearman correlation coefficient using the $\mathrm{R}$ statistical package. Protein or mRNA expression levels between different conditions or samples are assessed with the unpaired two-sided Student $t$-test and a significance level of 0.05 as cut-off. Calculation of standard errors of repeated experiments was performed using the error propagation rules.

\section{Results}

MYCN/c-MYC mRNA levels are inversely correlated with $D K K 3$ expression. Expression of DKK3 has previously been correlated to $M Y C N$ and $c-M Y C$ expression levels (here referred to as $M Y C N / c-M Y C){ }^{23,40,41}$ We confirmed the previously reported ${ }^{23}$ inverse relationship between $D K K 3$ expression levels and MYCN in a set of $12 \mathrm{NB}$ cell lines which were either MYCN amplified or MYCN single-copy (Fig. 1) and through analysis of their expression levels in an independent cohort of 101 primary untreated NB tumours $(r=$ $-0.30, p<0.01$ ) (Fig. 2). Analysis of the SHEP-Tet $21 \mathrm{~N}$ cell line with a tetracycline-regulable $M Y C N$ construct ${ }^{5,16}$ further confirmed this negative correlation, as shown by the decreased levels of DKK3 after MYCN induction (Fig. 3). 


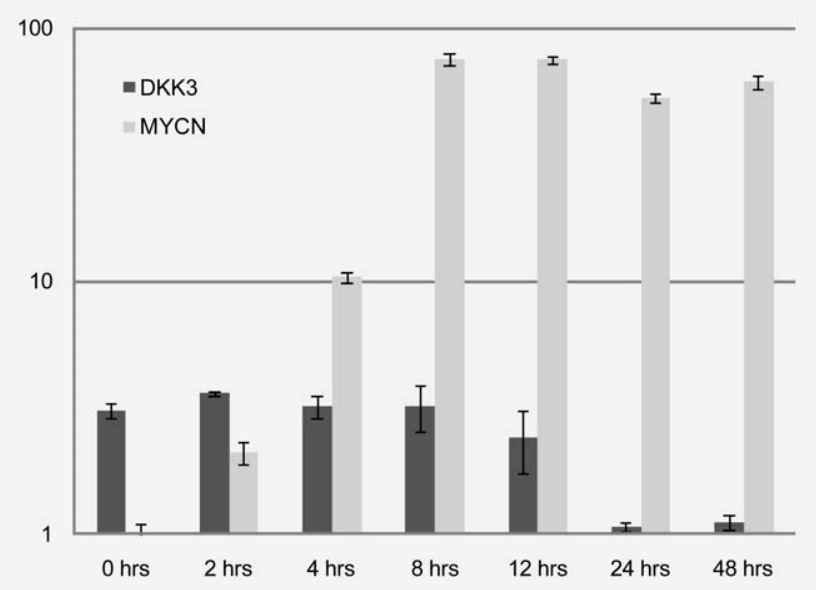

Figure 3. Inverse correlation between $D K K 3$ and $M Y C N$ using the SHEP-TET21N model system. Expression of DKK3 and MYCN are shown after $M Y C N$-induction at different timepoints in the SHEPTET21N model system (shown on $x$-axis). The $y$-axis is shown in a logaritmic scale.

Additionally, we evaluated the expression levels of $D K K 3$ and $M Y C N / c-M Y C$ in the NCI-60 cell line panel which contains cell lines of several tumour entities other then NB with elevated expression of MYCN or c-MYC. This analysis also yielded a significant negative correlation between $D K K 3$ and $M Y C N / c-M Y C$ (Spearman correlation $r=-0.51, p<0.01$ ). Altogether, these results confirm the tight relationship between MYCN and DKK3 expression levels.

\section{DKK3 expression levels are inversely correlated with the expression of members of the miR-17-92 cluster}

Using the SHEP-Tet21N model system, DKK3 downregulation only became apparent $24 \mathrm{hr}$ after $M Y C N$-induction suggesting indirect regulation of $D K K 3$ (Fig. 3). In view of the recently established role of the miR-17-92 cluster as an effector for indirect MYCN-induced downregulation of proteincoding genes, we hypothesized that this cluster might also regulate $D K K 3$. To investigate this possibility we first verified DKK3 mRNA expression following conditional upregulation of the miR-17-92 cluster in the SHEP-TR-miR-17-92 NB cell line. This indeed showed a significant downregulation of DKK3 transcripts (Fig. $4 a ; p=0.02$ after $72 \mathrm{hr}$ ) which was subsequently confirmed at the protein level (Fig. $4 b ; p<0.01$ after $72 \mathrm{hr}$ ), in keeping with the possibility that DKK3 is a direct target of one or more components from the miR-17-92 cluster.

Next, we performed an in silico search for putative miRNA target sites within the $3^{\prime}$ UTR of DKK3 using the TargetScan and DIANA microT prediction databases. Several members of the MYCN-activated miR-17-92 cluster, more specifically miR-19a, miR-19b and miR-92a, were predicted to target the $3^{\prime}$ UTR of DKK3 (Fig. $5 a$ ). We further looked for correlation between DKK3 expression and each of these three predicted miRNAs in a set of 101 primary untreated
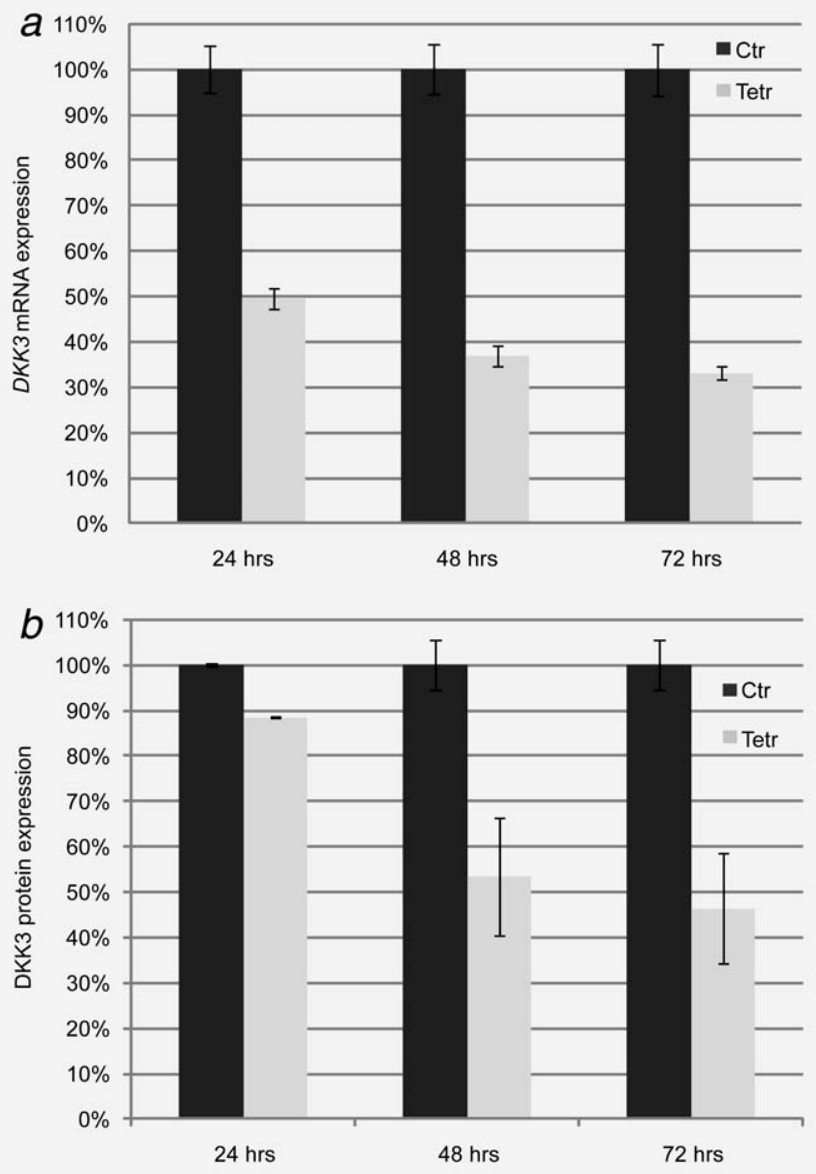

Figure 4. DKK3 expression levels using the SHEP-TR-miR-17-92 cell line. (a) mRNA expression levels of DKK3 after miR-17-92 induction in the SHEP-TR-miR-17-92 cell line. (b) Protein expression levels of DKK3 after miR-17-92 induction in the SHEP-TR-miR-17-92 cell line, measured using ELISA. Ctr = SHEP-TR-miR-17-92 cells treated with ethanol as control, Tetr $=$ SHEP-TR-miR-17-92 cells treated with tetracycline for induction of the miR-17-92 cluster.

NB tumours and all three miRNAs showed a significant inverse correlation with DKK3 (Supporting Information Table 1) which we could further confirm in the NCI-60 cell line collection (Supporting Information Table 2). These results suggest that the miR-17-92 cluster is the missing link between MYCN and DKK3.

\section{The DKK3 3'UTR is directly targeted by members of the miR-17-92 cluster}

To experimentally establish the direct role of the miR-17-92 cluster in DKK3 regulation, we tested the three miRNAs from the cluster with predicted putative binding sites in the 3'UTR of DKK3, i.e., miR-19a, miR-19b and miR-92a. Luciferase assays were performed in a cell line derived from colon carcinoma containing a hypomorphic mutation in Dicer (DLD1Dicer ${ }^{\text {hypo }}$ ). This cell line has a reduced amount of mature miRNA levels which makes the effect of pre-miR transfection higher than in non-mutated cell lines. Co- 


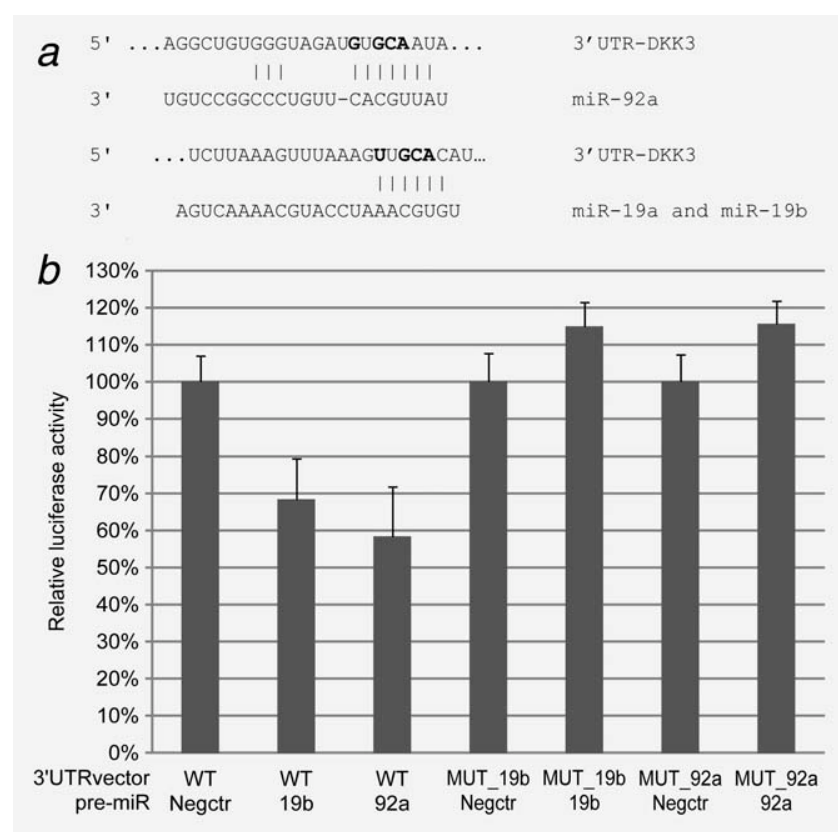

Figure 5. miRNA interaction with the $3^{\prime}$ UTR of DKK3. (a) Localisation of putative binding sites of miR-19 and miR-92a in the $3^{\prime} U T R$ of DKK3. The positions of mutations of the miRNA seed sequences (miR-19 or miR-92a) in the 3'UTR of DKK3 are shown in bold. (b) Relative luciferase activity for transfection of a wild type or mutated $3^{\prime} U T R$ DKK3 vector in combination with pre-miR-19a or pre-miR-92a. WT = wild type; MUT = mutated vector.

transfection of a selected pre-miR (pre-miR-19a, pre-miR-19b or pre-miR-92a) and a vector containing the $3^{\prime} \mathrm{UTR}$ of $D K K 3$ showed significant reduction for both pre-miR-19b $(p<$ $0.01)$ and pre-miR-92a $(p<0.01)$ indicating a direct interaction between these two miRNAs and the $3^{\prime} \mathrm{UTR}$ of DKK3 (Fig. 5b). Introduction of mutations in the miRNA seeds sites of miR-19b and miR-92a rescued the luciferase output which suggests that the effect of luciferase reduction depend on the presence of the miRNA seed sequences in the $3^{\prime}$ UTR (Fig. $5 b)$. No reduction in relative luciferase output could be observed upon repeated experiments for pre-miR-19a, thus excluding this miRNA as an effector of DKK3 expression (data not shown).

\section{miR-19b and miR-92a directly regulate $D K K 3$ expression}

Since both miR-19b and miR-92a interact with the $3^{\prime}$ UTR of $D K K 3$, we overexpressed each individual miRNA in the NB SHEP cell line using pre-miR mimics and evaluated the effect on both mRNA and protein DKK3 levels. As expected, for both miRNAs, DKK3 protein levels were shown to be significantly reduced after $72 \mathrm{hr}$ of pre-miR-19b or pre-miR-92a overexpression, respectively, $12 \%$ reduction $(p=0.02)$ and $58 \%$ reduction $(p<0.01$ ) (Fig. $6 a)$. Interestingly, only with pre-miR-92a transfections, reduced DKK3 mRNA levels were

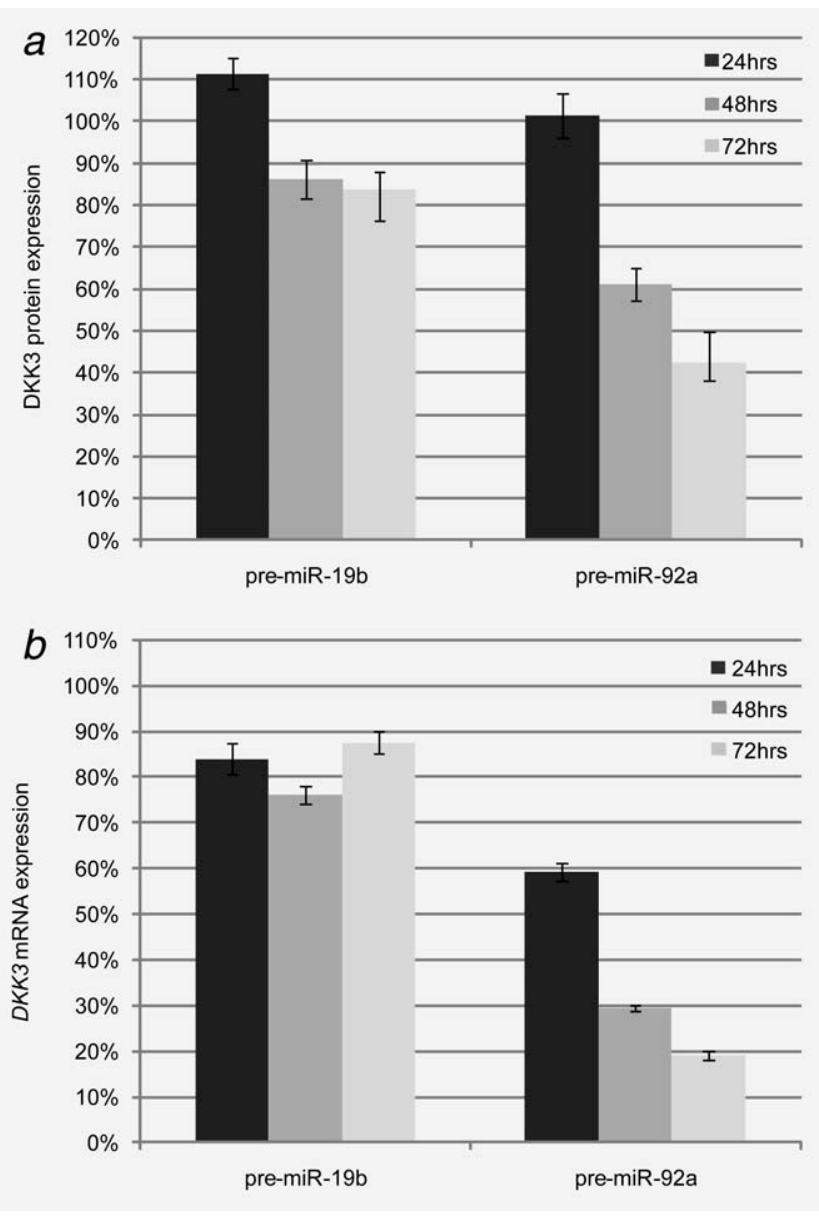

Figure 6. DKK3 levels after pre-miR-19b or pre-miR-92a transfection relative to control transfection. (a) Protein expression levels of DKK3 after pre-miR-19b or pre-miR-92a transfection of NB SHEP cells at 24,48 , and $72 \mathrm{hr}$ of transfection. (b) mRNA expression levels of $D K K 3$ after transfection of NB SHEP cells significantly reduced after pre-miR-92a overexpression. Pre-miR-19b overexpression did not result in reduction of $D K K 3$ mRNA levels.

observed (Fig. $6 b ; p=0.01$ ) indicating that each of these two components of the miR-17-92 cluster has a distinct effect on DKK3 regulation. To the best of our knowledge this is the first example of combined repressive effect on protein expression through targeting of mRNA stability and translation by different members of one miRNA cluster.

\section{Anti-miR-92a transfection restores DKK3 expression}

MYCN is induced in the SHEP MYCN-ER in vitro cell line model system by adding 4-OHT to the medium. As expected, transfection of this cell line with an anti-miR-neg ctr before and after MYCN-induction resulted in a significant $(p<$ 0.01 ) decrease in DKK3 levels upon MYCN-induction (Supporting Information Fig. 1). Anti-miR-92a transfection after MYCN-induction was, however, able to restore the $D K K 3$ levels. The increase of $D K K 3$ levels compared to the non- 
induced cells line is possibly explained by the fact that antimiR-92a transfection not only reduces the MYCN-induced miR-92a levels but additionally reduces the endogenous miR92a levels.

In line with our hypothesis, these results exclude a significant contribution in DKK3 regulation through other $\mathrm{MYCN}$ regulated genes.

\section{Discussion}

The MYCN transcription factor is known to regulate a broad range of genes affecting various cellular functions implicated in normal development and cancer. ${ }^{2}$ Studies of MYCN-regulated genes have mainly focussed on direct upregulated genes. A few studies also demonstrate a role for MYCN in downregulation of genes but these mechanisms of are less well understood. At least for some target genes, MYCN is recruited to the promoter by the DNA binding protein MIZ1 followed by epigenetic silencing, in part due to concomitant action of DNMT3a. ${ }^{17}$ Recently, miRNA-mediated silencing has emerged as an alternative mechanism for MYCN-driven downregulation of gene expression. Several MYCN-upregulated miRNAs were identified and have provided evidence that miRNA activation contributes to widespread MYCNinduced mRNA repression. ${ }^{6,38}$ Using in silico analyses based on predicted seeds in the $3^{\prime} \mathrm{UTR}$ of downregulated mRNAs and predicted MYCN downregulated genes from the MYCNot database (http://medgen.ugent.be/MYCNot), we provided strong evidence that activated miRNA expression could serve as a mechanism for such a MYCN-mediated mRNA repression. In this study, we present experimental evidence for indirect downregulation of DKK3 through direct $\mathrm{MYCN}$ induced transcriptional activation of the miR-17-92 cluster.

DKK3 is a member of the Dickkopf family together with DKK1, 2, 4 and Soggy. While DKK1, 2 and 4 have a wellestablished role as WNT-inhibitors, the functions of DKK3 and its homologue Soggy are less firmly established, although recent studies on DKK3 also provide evidence for a role in the canonical WNT pathway. ${ }^{35,41}$ In the study of Tudzarova and colleagues, DKK3 is shown to interact with the betaTrCP which is a negative regulator of beta-catenin, a key component in the WNT signaling pathway. DKK3 has been proposed as a tumour suppressor given the observed reduced expression levels in several tumour entities and tumour suppressing effects upon overexpression in certain cancer cell types. These observations are in line with reduced expression following MYCN overexpression but a relation between DKK3 and WNT signaling could not be established in NB.

Here, we elucidate the mechanism of downregulation of $D K K 3$ as a result of post-transcriptional regulation through the miR-17-92 cluster. Evidence for such an interaction was obtained for two of the six members of the cluster, miR-19b and miR-92a. Although pre-miR-19a was also predicted to target the $3^{\prime} \mathrm{UTR}$ of $D K K 3$, we could not observe a reduced luciferase output nor reduced DKK3 mRNA or protein levels after transient transfection (data not shown). For miR-19b and miR-92a, reduced DKK3 protein levels were detected upon a 72-hr overexpression in the MYCN single-copy SHEP NB cell line. Interestingly, decrease of the mRNA levels were only noted after overexpression of miR-92a which could subsequently be restored upon anti-miR-92a transfection. These findings suggest that different members of the same miRNA cluster regulate target gene expression on distinctive levels by effecting mRNA stability or protein translation.

In this study we focussed on miR-17-92 and its components given its direct regulation through MYCN. It should be noted however that other miRNAs, such as members of the miR-17-92 paralog clusters, miR-106b-25 and miR-106a-363, are also predicted to target $D K K 3$.

In addition to the present report, our recent investigation of putative miR-17-92 targets also demonstrated regulation of several components of the TGF- $\beta$ pathway. ${ }^{38}$ Together with other previously described target genes, this most probably only represents a small proportion of all bona fide target genes which are regulated by this important oncogenic miRNA cluster. It will be of interest to investigate which of these miRNAs and subsequent target genes play a crucial role in the tumour phenotype as a prelude to design new therapies. Also, given the tight regulation of DKK3 and its presumed role as tumour suppressor gene, further investigations towards unravelling the function of this protein are warranted. One possible line of further investigation is the role of DKK3 in relation to control of the so-called origin activation checkpoint, as described in a recent paper by Tudzarova et al. ${ }^{41}$ In this study it is shown that DKK3 is a direct target of TP53 which is activated as a consequence of disruption of the origin activation checkpoint leading to G1/S arrest. Reduction of DKK3 levels through MYCN could very well fit with one of the major functions of MYCN as driver of proliferation and progression through the cell cycle.

In conclusion, this study shows a direct regulation of DKK3 mRNA and protein levels by members of the highly oncogenic miR-17-92 cluster. As MYCN has been shown to induce this cluster and because MYCN amplification is a marker for poor prognosis, these results suggest a critical role for DKK3 as tumour suppressor in NB development. Further investigation of the biological function of DKK3 and its role as tumour suppressor gene in NB pathogenesis are therefore of great interest in the light of development of novel therapeutic strategies.

\section{Acknowledgements}

The authors thank Fanny De Vloed for her excellent practical work. S.D.B. is supported by an "Emmanuel van der Schueren" grant from the "Vlaamse Liga tegen Kanker", P.M. by the Ghent University Research Fund (BOF 01D31406), F.P.T. and K.D.P. are postdoctoral fellows of the Research Foundation Flanders (F.W.O.) and F.W. was funded by the Bundesministerium für Bildung und Forschung (BMBF, NGFNPlus). The funders had no role in study design, data collection and analysis, decision to publish, or preparation of the manuscript. 


\section{References}

1. Maris JM. Recent advances in neuroblastoma. N Engl J Med 2010;362: 2202-11.

2. Bell E, Chen L, Liu T, Marshall GM, Lunec J, Tweddle DA. MYCN oncoprotein targets and their therapeutic potential. Cancer Lett 2010;293:144-57.

3. Luscher B. Function and regulation of the transcription factors of the Myc/Max/Mad network. Gene 2001;277:1-14.

4. Weiss WA, Aldape K, Mohapatra G, Feuerstein BG, Bishop JM. Targeted expression of MYCN causes neuroblastoma in transgenic mice. EMBO J 1997;16: 2985-95.

5. Lutz W, Stohr M, Schurmann J, Wenzel A, Lohr A, Schwab M. Conditional expression of N-myc in human neuroblastoma cells increases expression of alpha-prothymosin and ornithine decarboxylase and accelerates progression into S-phase early after mitogenic stimulation of quiescent cells. Oncogene 1996;13:803-12.

6. Schulte JH, Horn S, Otto T, Samans B, Heukamp LC, Eilers UC, Krause M, Astrahantseff K, Klein-Hitpass L, Buettner $\mathrm{R}$, Schramm A, Christiansen $\mathrm{H}$, et al. MYCN regulates oncogenic MicroRNAs in neuroblastoma. Int J Cancer 2008;122: 699-704.

7. Tang XX, Zhao H, Kung B, Kim DY, Hicks SL, Cohn SL, Cheung NK, Seeger RC, Evans AE, Ikegaki N. The MYCN enigma: significance of MYCN expression in neuroblastoma. Cancer Res 2006;66: 2826-33.

8. Cetinkaya C, Hultquist A, Su Y, Wu S, Bahram F, Pahlman S, Guzhova I, Larsson LG. Combined IFN-gamma and retinoic acid treatment targets the N-Myc/Max/ Mad1 network resulting in repression of $\mathrm{N}-\mathrm{Myc}$ target genes in MYCN-amplified neuroblastoma cells. Mol Cancer Ther 2007;6:2634-41.

9. Nakamura M, Matsuo T, Stauffer J, Neckers L, Thiele CJ. Retinoic acid decreases targeting of p27 for degradation via an N-myc-dependent decrease in p27 phosphorylation and an N-mycindependent decrease in Skp2. Cell Death Differ 2003;10:230-9.

10. Kang JH, Rychahou PG, Ishola TA, Qiao J, Evers BM, Chung DH. MYCN silencing induces differentiation and apoptosis in human neuroblastoma cells. Biochem Biophys Res Commun 2006;351:192-7.

11. Nara K, Kusafuka T, Yoneda A, Oue T, Sangkhathat S, Fukuzawa M. Silencing of MYCN by RNA interference induces growth inhibition, apoptotic activity and cell differentiation in a neuroblastoma cell line with MYCN amplification. Int J Oncol 2007;30:1189-96.
12. Woo CW, Tan F, Cassano H, Lee J, Lee $\mathrm{KC}$, Thiele CJ. Use of RNA interference to elucidate the effect of MYCN on cell cycle in neuroblastoma. Pediatr Blood Cancer 2008;50:208-12.

13. Mac SM, D'Cunha CA, Farnham PJ. Direct recruitment of $\mathrm{N}$-myc to target gene promoters. Mol Carcinog 2000;29:76-86.

14. Shohet JM, Hicks MJ, Plon SE, Burlingame SM, Stuart S, Chen SY, Brenner MK, Nuchtern JG. Minichromosome maintenance protein MCM7 is a direct target of the MYCN transcription factor in neuroblastoma. Cancer Res 2002;62: 1123-8.

15. Slack A, Chen Z, Tonelli R, Pule M, Hunt L, Pession A, Shohet JM. The p53 regulatory gene MDM2 is a direct transcriptional target of $\mathrm{MYCN}$ in neuroblastoma. Proc Natl Acad Sci USA 2005;102:731-6.

16. Westermann F, Muth D, Benner A, Bauer T, Henrich KO, Oberthuer A, Brors B, Beissbarth T, Vandesompele J, Pattyn F, Hero B, Konig R, et al. Distinct transcriptional MYCN/c-MYC activities are associated with spontaneous regression or malignant progression in neuroblastomas. Genome Biol 2008;9:R150.

17. Iraci N, Diolaiti D, Papa A, Porro A, Valli E, Gherardi S, Herold S, Eilers M, Bernardoni R, Della Valle G, Perini G. A SP1/MIZ1/MYCN repression complex recruits $\mathrm{HDAC1}$ at the TRKA and p75NTR promoters and affects neuroblastoma malignancy by inhibiting the cell response to NGF. Cancer Res 2011; 71:404-12.

18. Bell E, Lunec J, Tweddle DA. Cell cycle regulation targets of MYCN identified by gene expression microarrays. Cell Cycle 2007;6:1249-56.

19. Glinka A, Wu W, Delius H, Monaghan AP, Blumenstock C, Niehrs C. Dickkopf-1 is a member of a new family of secreted proteins and functions in head induction. Nature 1998;391:357-62.

20. Kawano Y, Kypta R. Secreted antagonists of the Wnt signalling pathway. J Cell Sci 2003;116:2627-34.

21. Mao B, Niehrs C. Kremen2 modulates Dickkopf2 activity during Wnt/LRP6 signaling. Gene 2003;302:179-83.

22. Wu W, Glinka A, Delius H, Niehrs C. Mutual antagonism between dickkopf1 and dickkopf2 regulates Wnt/beta-catenin signalling. Curr Biol 2000;10:1611-14.

23. Koppen A, Ait-Aissa R, Koster J, Ora I, Bras J, van Sluis PG, Caron H, Versteeg R, Valentijn LJ. Dickkopf-3 expression is a marker for neuroblastic tumor maturation and is down-regulated by MYCN. Int J Cancer 2008;122:1455-64.
24. Revet I, Huizenga G, Koster J, Volckmann $\mathrm{R}$, van Sluis P, Versteeg R, Geerts D. MSX1 induces the Wnt pathway antagonist genes DKK1, DKK2, DKK3, and SFRP1 in neuroblastoma cells, but does not block Wnt3 and Wnt5A signalling to DVL3. Cancer Lett 2010;289:195-207.

25. Kobayashi K, Ouchida M, Tsuji T, Hanafusa H, Miyazaki M, Namba M, Shimizu N, Shimizu K. Reduced expression of the REIC/Dkk-3 gene by promoterhypermethylation in human tumor cells. Gene 2002;282:151-8.

26. Lodygin D, Epanchintsev A, Menssen A, Diebold J, Hermeking H. Functional epigenomics identifies genes frequently silenced in prostate cancer. Cancer Res 2005;65:4218-27.

27. Roman-Gomez J, Jimenez-Velasco A, Agirre X, Castillejo JA, Navarro G, Barrios M, Andreu EJ, Prosper F, Heiniger A, Torres A. Transcriptional silencing of the Dickkopfs-3 (Dkk-3) gene by CpG hypermethylation in acute lymphoblastic leukaemia. Br J Cancer 2004;91: 707-13.

28. Tsuji T, Nozaki I, Miyazaki M, Sakaguchi M, Pu H, Hamazaki Y, Iijima O, Namba M. Antiproliferative activity of REIC/Dkk-3 and its significant down-regulation in nonsmall-cell lung carcinomas. Biochem Biophys Res Commun 2001;289:257-63.

29. Abarzua F, Sakaguchi M, Takaishi M, Nasu Y, Kurose K, Ebara S, Miyazaki M, Namba M, Kumon H, Huh NH. Adenovirusmediated overexpression of REIC/Dkk-3 selectively induces apoptosis in human prostate cancer cells through activation of c-Jun-NH2-kinase. Cancer Res 2005;65: 9617-22.

30. Hoang BH, Kubo T, Healey JH, Yang R, Nathan SS, Kolb EA, Mazza B, Meyers PA, Gorlick R. Dickkopf 3 inhibits invasion and motility of Saos-2 osteosarcoma cells by modulating the Wnt-beta-catenin pathway. Cancer Res 2004;64:2734-9.

31. Hsieh SY, Hsieh PS, Chiu CT, Chen WY. Dickkopf-3/REIC functions as a suppressor gene of tumor growth. Oncogene 2004;23: 9183-9.

32. Kawano Y, Kitaoka M, Hamada Y, Walker MM, Waxman J, Kypta RM. Regulation of prostate cell growth and morphogenesis by Dickkopf-3. Oncogene 2006;25:6528-37.

33. Kuphal S, Lodermeyer S, Bataille F, Schuierer M, Hoang BH, Bosserhoff AK. Expression of Dickkopf genes is strongly reduced in malignant melanoma. Oncogene 2006;25:5027-36.

34. Kurose K, Sakaguchi M, Nasu Y, Ebara S, Kaku H, Kariyama R, Arao Y, Miyazaki M, Tsushima T, Namba M, Kumon H, Huh NH. Decreased expression of REIC/Dkk-3 
in human renal clear cell carcinoma. J Urol 2004;171:1314-8.

35. Lee EJ, Jo M, Rho SB, Park K, Yoo YN, Park J, Chae M, Zhang W, Lee JH. Dkk3, downregulated in cervical cancer, functions as a negative regulator of beta-catenin. Int J Cancer 2009;124: 287-97.

36. Tsuji T, Miyazaki M, Sakaguchi M, Inoue Y, Namba M. A REIC gene shows down-regulation in human immortalized cells and human tumor-derived cell lines. Biochem Biophys Res Commun 2000;268: $20-4$.
37. Mestdagh P, Fredlund E, Pattyn F, Schulte JH, Muth D, Vermeulen J, Kumps C, Schlierf S, De Preter K, Van Roy N, Noguera R, Laureys G, et al. MYCN/cMYC-induced microRNAs repress coding gene networks associated with poor outcome in MYCN/c-MYC-activated tumors. Oncogene 2010;29: 1394-404.

38. Mestdagh $P$, Bostrom AK, Impens $F$, Fredlund E, Van Peer G, De Antonellis P, von Stedingk K, Ghesquiere B, Schulte S, Dews M, Thomas-Tikhonenko A, Schulte JH, et al. The miR-17-92 microRNA cluster regulates multiple components of the TGF-beta pathway in neuroblastoma. Mol Cell 2010;40:762-73.

39. Lefever S, Vandesompele J, Speleman F, Pattyn F. RTPrimerDB: the portal for realtime PCR primers and probes. Nucleic Acids Res 2009;37:D942-5.

40. Clevers H. Wnt/beta-catenin signaling in development and disease. Cell 2006;127: 469-80.

41. Tudzarova S, Trotter MW, Wollenschlaeger A, Mulvey C, Godovac-Zimmermann J, Williams GH, Stoeber K. Molecular architecture of the DNA replication origin activation checkpoint. EMBO J 2010;29: 3381-94. 